\title{
Clinical testing for the nevoid basal cell carcinoma syndrome in a DNA diagnostic laboratory
}

Roger D. Klein, MD, Daniel J. Dykas, AS, and Allen E. Bale, MD

\begin{abstract}
Purpose: This study determines which clinical features predict positive test results among samples submitted for DNA-based diagnostic nevoid basal cell carcinoma syndrome (NBCCS) testing, and further defines the mutational spectrum of the $\mathrm{PTCH}$ gene. Methods: DNA was extracted from peripheral blood leukocytes, and polymerase chain reaction products from exons 1 to 23 of the $P T C H$ gene were directly sequenced. Pedigree phenotypic information was obtained by written questionnaire. Results: Among 106 presumably unrelated pedigrees, 44 independent mutations were found in 47 families. There were 11 nonsense mutations; 1 in-frame deletion; 17 deletions, 6 insertions, and 1 deletion-insertion that generated frameshifts; 5 splice-site mutations; 1 in-frame duplication; and 2 presumptive missense mutations. Twenty-seven of 46 pedigrees $(58.7 \%)$ with two or more typical radiographic or pathologic features of NBCCS tested positive for PTCH mutations. Of these, 26 had jaw cysts in combination with other characteristics or neoplasms including basal cell carcinomas, palmar pits, skeletal abnormalities, ocular abnormalities, medulloblastomas, cardiac or ovarian fibromas, calcification of the falx cerebri, polydactyly, cleft lip and/or palate, and agenesis of the corpus callosum or other central nervous system malformations. None of the 13 pedigrees solely affected by multiple or early-onset basal cell carcinomas and none of the four pedigrees with jaw cysts alone had PTCH mutations. Conclusions: Pedigrees with multiple features of NBCCS were most likely to test positive for PTCH mutations. Pedigrees with multiple or early-onset basal cell carcinomas without other features of the disease did not test positive for PTCH mutations. Genet Med 2005:7(9):611-619.
\end{abstract}

Key Words: nevoid basal cell carcinoma syndrome, NBCCS, basal cell nevus syndrome, PTCH, Gorlin syndrome

Nevoid basal cell carcinoma syndrome (NBCCS), also known as Gorlin syndrome or basal cell nevus syndrome (BCNS), is an autosomal dominant disorder that is characterized by a predisposition to neoplasms and developmental abnormalities. NBCCS has essentially complete penetrance and highly variable expressivity both within and among families. ${ }^{1}$ Systematic assessment of patients with NBCCS has demonstrated an excess of multiple basal cell carcinomas, odontogenic keratocysts, palmar and plantar epidermal pits, calcification of the falx cerebri, spine and rib anomalies, relative macrocephaly, frontal bossing, facial milia, ocular malformations, medulloblastomas, cleft lip and/or palate, and other neoplasms and developmental malformations in the syndrome..$^{2-5}$ Basal cell carcinomas in patients with NBCCS tend to occur at younger ages and in greater numbers than in patients who have sporadic basal cell tumors. ${ }^{2-4}$ The prevalence of NBCCS has been estimated to be from 1 in $57,000^{6}$ to 1 in $164,000 .^{3}$

\footnotetext{
From the DNA Diagnostic Laboratory, Department of Genetics, Yale University School of Medicine, New Haven, Connecticut.

Roger D. Klein, MD, DNA Diagnostic Laboratory, Yale University School of Medicine, Department of Genetics, SHM I-321, 333 Cedar Street, New Haven, CT 06510.

Received: May 24, 2005.

Accepted: August 8, 2005.
}

DOI: 10.1097/01.gim.0000182879.57182.b4
NBCCS is caused by inactivating mutations in the human homolog of the Drosophila segment-polarity gene patched $(P T C H){ }^{7,8}$ Nonsense, frameshift, in-frame deletions, splicesite, and missense mutations all have been described in the syndrome. Most mutations are expected to result in the production of truncated proteins. Reported mutations are scattered throughout the coding region. There have been few recurrent mutations and no genotype-phenotype correlations described. ${ }^{9}$ Tumors in the syndrome arise with loss of both copies of $P T C H$ in accordance with the two-hit model of Knudson. ${ }^{10}$ Typically, a germline point mutation or small deletion in $\mathrm{PTCH}$ combines with somatic loss of the wild-type $P T C H$ allele through gross chromosomal mechanisms. Sporadic basal cell carcinomas also arise with loss of $P T C H .7,8,11-13$ In addition, some randomly occurring developmental abnormalities, such as bifid ribs and jaw cysts, may be generated through a two-hit mechanism. ${ }^{14}$ Other generalized or symmetric NBCCS developmental abnormalities such as overgrowth, macrocephaly, and facial dysmorphology are believed to be the product of $P T C H$ haploinsufficiency.

PTCH has been mapped to chromosome 9q22.3. The $34 \mathrm{~kb}$ gene contains 24 exons and encodes a 1447 amino acid glycoprotein. The $P T C H$ protein has 12 transmembrane domains, with two extracellular loops that bind the "Hedgehog" signaling proteins. The region of the gene that includes transmembrane domains 2 through 6 is homologous to the sterol sensing 
domains of other proteins. ${ }^{15}$ The most widely expressed Hedgehog protein in mammals appears to be "Sonic" Hedgehog. In its unbound state, $P T C H$ constitutively inhibits the activity of an affiliated $G$ protein-coupled-like receptor referred to as Smoothened. Hedgehog binding releases the inhibitory action of PTCH on Smoothened, producing downstream activation of the Hedgehog pathway. ${ }^{7,8,15-18}$ Loss of function of both PTCH alleles causes over-activation of the Hedgehog pathway, with consequent dysregulated cellular growth and differentiation.

Although patients with NBCCS can often be diagnosed on the basis of clinical findings, genetic tests that identify PTCH mutations are useful in many situations. This testing can be used to confirm the diagnosis in patients in whom NBCCS is suspected, to establish the diagnosis in patients at risk for the disease and for family planning, prenatal, and preimplantation diagnostic purposes. Because patients with NBCCS are predisposed to neoplastic disease, patients who have or are at risk for the syndrome are advised to undergo regular surveillance. Monitoring for jaw cysts and preventive activities such as avoidance of sunlight and radiation therapy are also recommended. ${ }^{4}$ In families in whom a mutation has been identified, individuals who test negative for that mutation can avoid the stress and expense of monitoring, and can be reassured that they will not pass on NBCCS to their progeny. Because as many as $50 \%$ of patients with NBCCS appear to have new mutations, PTCH mutation testing is helpful in diagnosing children who have features suggestive of NBCCS but no family history of the syndrome.

In this study we report the results of clinical PTCH testing in a DNA diagnostic laboratory, including data that can assist patient counseling and help direct appropriate test ordering. We also describe 36 previously unreported mutations and three variants of unknown significance.

\section{MATERIALS AND METHODS}

\section{Participants}

From February 1997 to May 2005, blood samples from 143 individuals representing 106 pedigrees were tested in the Yale DNA Diagnostic Laboratory for mutations in $\mathrm{PTCH}$.

\section{Clinical surveys}

A request for clinical information, including patient and family history, was included as a part of our laboratory's sample requisition form. In addition, a one-page patient and family history questionnaire was mailed to the referring physician or genetic counselor for each patient about whom sufficient clinical information did not accompany the submitted blood sample. Physicians and/or genetic counselors were questioned specifically about the presence of basal cell carcinomas; palmar pits; jaw cysts; calcification of the falx cerebri; rib or spine abnormalities; medulloblastoma; cleft lip and/or palate; polydactyly; ovarian fibroma; cardiac fibroma; cataract, coloboma, and other ocular anomalies; and agenesis of the corpus callosum and other neurologic abnormalities. These features were selected because of their frequencies in NBCCS, relative ease of objective assessment, and/or discriminatory ability. Blank space in the questionnaire was also provided for additional information and family history.

\section{Mutation testing}

Genomic DNA from peripheral blood leukocytes was extracted using standard methods. ${ }^{19,20}$ DNA sequence abnormalities were identified by polymerase chain reaction amplification and sequencing of the coding exons of the PTCH gene, including three alternate splice forms of exon 1 , and exons 2 to 23 with corresponding intron-exon junctions (primers listed in Table 1). All mutations were confirmed by bidirectional sequencing or by the use of restriction enzyme digests for mutations that were predicted to alter restriction sites. Potential splice-site mutations were analyzed using the splice-site predictor programs at http://www.fruitfly.org or http://125. itba.mi.cnr.it/ webgene/wwwspliceview.html. In-frame deletions and missense mutations were judged probably deleterious if they were not found in 100 unaffected individuals, altered highly conserved amino acids, and segregated with the disease in those cases for whom more than one affected family member was available for study. Once a disease-causing mutation was identified in a family, additional family members were tested by direct sequencing or restriction enzyme digestion.

\section{Statistical analysis}

The statistical significance of differences in the proportions of patients testing positive for $\mathrm{PTCH}$ mutations among various phenotypes was assessed using the chi-square test or Fisher exact test. The mean ages of patients testing positive and negative for $P T C H$ mutations were compared using a two-sided $t$ test.

\section{RESULTS}

Among 106 presumably unrelated pedigrees, 44 independent mutations were found in 47 kindreds (Table 2). Fifty-six families tested negative for $\mathrm{PTCH}$ mutations. In three families variants of unknown significance were identified. These variants included nonsynonymous base pair substitutions in exons 6 and 19, and a $\mathrm{C} \rightarrow \mathrm{A}$ change in nucleotide 94 of exon 1 (GenBank U43148.1), which to date has not been shown to be coding. One previously reported mutation in codon 135 of exon 3, CGA $\rightarrow$ TGA, was seen in four pedigrees. Four mutations were intronic splice-site mutations. There were 11 nonsense mutations; 1 in-frame deletion; 17 deletions, 6 insertions, and 1 deletion-insertion that generated frameshifts; 5 splice-site mutations; 1 in-frame duplication; and 2 presumptive missense mutations.

A presumed missense mutation G509D has been reported in an affected Japanese patient. There is strong evidence that this amino acid substitution is a causative mutation based on the report of another putative missense mutation in this codon, G509V; the absence of the G509D mutation in the 105 other 
Table 1

$P T C H$ polymerase chain reaction and sequencing primers

\begin{tabular}{|c|c|c|}
\hline Exon & Forward & Reverse \\
\hline 1 & $\begin{array}{l}\text { GAAGGCGAGCACCCAGAC }^{a} \\
\text { GGGTTCATTGTGTTTACGAG }^{a}\end{array}$ & $\begin{array}{l}\text { TCTTTCCCTCCTCTCCTTCT } \\
\text { GAGCTGAATTAGGAAGTGG }\end{array}$ \\
\hline $1 \mathrm{~A}$ & GCTATGGAAATGCGTCGG & CAGTCCTGCTCTGTCCATCA $^{a}$ \\
\hline $1 \mathrm{~B}$ & TCTTCCGCGAACTGGATGTG $^{a}$ & GGGGCGATCCCAAAGAGTTAG $^{a}$ \\
\hline 2 & GTGGCTGAGAGCGAAGTTTC & TCTCTATCAACCGCGAGGAG $^{a}$ \\
\hline 3 & CTATTGTGTATCCAATGGCAGG $^{a}$ & ATTAGTAGGTGGACGCGGC \\
\hline 4 & AGAGAAATTTTTGTCTCTGCTTTTCA & TAAGGCACACTACTGGGGTG $^{a}$ \\
\hline 5 & GCAAAAATTTCTCAGGAACACC $^{a}$ & TGGAACAAACAATGATAAGCAA \\
\hline 6 & CCTACAAGGTGGATGCAGTG $^{a}$ & TTTGCTCTCCACССTTCTGA \\
\hline 7 & GTGACCTGCCTACTAATTCCC ${ }^{a}$ & GGCTAGCGAGGATAACGGTTTA \\
\hline 8 & GAGGCAGTGGAAACTGCTTC $^{a}$ & TTGCATAACCAGCGAGTCTG \\
\hline 9 & GTGCTGTCGAGGCTTGTG ${ }^{a}$ & AGAAGCAGGAGCAGTCATGG \\
\hline 10 & TTCGGCTTTTGTTCTGTGC ${ }^{a}$ & CCGGTGGCATTTGTCAAC \\
\hline 11 & GTGTTAGGTGCTGGTGGCA $^{a}$ & CTTAGGAACAGAGGAAGCTG \\
\hline 12 & GACCATGTCCAGTGCAGCTC $^{a}$ & CGTTCAGGATCACCACAGCC \\
\hline 13 & AGTCCTCTGATTGGGCGGAG $^{a}$ & CCATTCTGCACCCAATCAAAAG \\
\hline 14 & AAAATGGCAGAATGAAAGCACC ${ }^{a}$ & CTGATGAACTCCAAAGGTTCTG \\
\hline 15 & GACAGCTTCTCTTTGTCCAG & ACGCAAAAGACCGAAAGGACGA $a$ \\
\hline 16 & AGGGTCCTTCTGGCTGCGAG ${ }^{a}$ & GCTGTCAAGCAGCCTCCAC \\
\hline 17 & AACCССАTTCTCAAAGGCСTCTGTC ${ }^{a}$ & CACCTCTGTAAGTTCCCTGACCT $^{a}$ \\
\hline 18 & AACTGTGATGCTCTTCTACCCTGG ${ }^{a}$ & AAACTTCCCGGCTGCAGAAAGA \\
\hline 19 & TTTGATCTGAACCGAGGACACC ${ }^{a}$ & CAAACAGAGCCAGAGGAAATCG \\
\hline 20 & TAGGACAGAGCTGAGCATTTACC ${ }^{a}$ & CTAAAGTGCTGGGATTACAGGC \\
\hline 21 & TGTTCCCGTTTCCTCTTG ${ }^{a}$ & GCACAGGAAACACAGCATTC $^{a}$ \\
\hline 22 & CAGGTAAATGGACAAGAAC ${ }^{a}$ & CTGTGTGATGTGCTGCTC \\
\hline \multirow[t]{2}{*}{23} & ССТТСТААСССАСССТСАССС & TTCCCCAAGGATGACAAAGCTTG \\
\hline & TTTTGAAATTTCTACTGAAGGGCAT ${ }^{b}$ & GAGGCAGAAGCCGTCACAG $^{b}$ \\
\hline
\end{tabular}

${ }^{a}$ Primer also used for sequencing.

${ }^{b}$ Primer only used for sequencing.

pedigrees that we clinically tested for NBCCS; a failure to detect the mutation in 108 unrelated, unaffected Japanese chromosomes and 84 African American chromosomes; the location of this glycine residue in a critical sterol sensing domain (fourth transmembrane domain); and its high evolutionary conservation..$^{21}$ The second apparent missense mutation identified in our study population, $\mathrm{P593R}$, also is located within the PTCH sterol sensing domain in the sixth transmembrane domain.

Most mutations were unique and scattered throughout the coding sequence in a manner that was approximately proportional to exon size (Table 3). No mutations were found in eight exons, including the three alternate splice forms of exon 1 and exon 23, the largest coding exon (540 base pair coding region). The largest number of mutations was found in exon $2,{ }^{6}$ which contains 193 base pairs. Mutations involving the same six codons of exon 2 are described in the PTCH Mutation Database (http://www.cybergene.SE/cgi-bin/w3-msql/ptchbase/ index.html). This database also reports a limited number of mutations in exon 23. Although a predominance of exon 2 mutations does not appear to be present in the PTCH Mutation Database, overall our data seem to be consistent with the mutation spectrum reported there. The reason for an apparent relative paucity of mutations in exon 23 in both sets of data is unclear, and it is difficult to draw meaningful conclusions from the fairly small number of mutations that have been reported.

Detailed clinical information was obtained for 28 pedigrees that tested positive for $P T C H$ mutations and 44 pedigrees that tested negative, a statistically significant difference $(P=.009)$. This discrepancy may reflect privacy concerns among some providers who were reluctant to retrospectively provide clinical information about pedigrees with known disease-causing 
Klein et al.

Table 2

Mutations in nevoid basal cell carcinoma syndrome pedigrees

\begin{tabular}{|c|c|c|}
\hline Exon/Intron & Codon & Codon Change \\
\hline \multicolumn{3}{|l|}{ Nonsense Mutations } \\
\hline \multirow[t]{2}{*}{2} & 84 & $\mathrm{CAG} \rightarrow \mathrm{TAG}^{\mathrm{a}}(\mathrm{Gln} \rightarrow$ Stop $)$ \\
\hline & 127 & GAG $\rightarrow$ TAG $($ Glu $\rightarrow$ Stop $)$ \\
\hline 3 & 135 & $\mathrm{CGA} \rightarrow \mathrm{TGA}^{\mathrm{a}, \mathrm{b}}(\operatorname{Arg} \rightarrow$ Stop $)$ \\
\hline \multirow[t]{2}{*}{5} & 221 & GAA $\rightarrow$ TAA $($ Glu $\rightarrow$ Stop $)$ \\
\hline & 241 & TTA $\rightarrow$ TGA $($ Leu $\rightarrow$ Stop $)$ \\
\hline \multirow[t]{3}{*}{6} & 256 & TTG $\rightarrow$ TAG $($ Leu $\rightarrow$ Stop $)$ \\
\hline & 274 & $\mathrm{CAA} \rightarrow$ TAA $(\mathrm{Gln} \rightarrow$ Stop $)$ \\
\hline & 313 & TCA $\rightarrow$ TGA (Ser $\rightarrow$ Stop $)$ \\
\hline 8 & 387 & $\mathrm{TGG} \rightarrow \mathrm{TAG}^{\mathrm{a}}(\mathrm{Trp} \rightarrow$ Stop $)$ \\
\hline 17 & 926 & $\mathrm{TGG} \rightarrow \mathrm{TGA}^{\mathrm{a}}(\mathrm{Trp} \rightarrow$ Stop $)$ \\
\hline 19 & 1093 & GGA $\rightarrow$ TGA (Gly $\rightarrow$ Stop $)$ \\
\hline
\end{tabular}

In-Frame Deletions ${ }^{c}$

$437-443$

In-Frame Duplications ${ }^{c}$

$367-369$

Deletions Creating Frameshifts

86
$88-89$
110
292
342
403
447,448
472,473
568
770
812
858
861
975
1094
1122
1124

1180,1181

21

Insertions Creating Frameshifts

2

6

7

10

13

15

Splice-Site Mutations ${ }^{\mathrm{d}}$

10

10

11

14

15

Probable Missense Mutations ${ }^{c}$

11

13

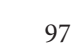

\begin{abstract}
del CT ${ }^{\mathrm{a}}$
del TTTA

del G

del A

del T

del $\mathrm{AT}^{\mathrm{a}}$

del TACT

del TGGC

del C

del C

del C

del C

del $\mathrm{G}^{a}$

del C

del G

$\operatorname{del~\mathrm {AT}^{a}}$

del C
\end{abstract}

delATATinsTCC

ins A

ins $\mathrm{A}$

ins $\mathrm{A}$

ins TG

ins $\mathrm{T}$

ins $\mathrm{T}$

$\mathrm{G} \rightarrow \mathrm{C}$

$\mathrm{CAG} \rightarrow \mathrm{CAA}$

$\mathrm{G} \rightarrow \mathrm{A}$

$\mathrm{A} \rightarrow \mathrm{G}$

$\mathrm{T} \rightarrow \mathrm{C}$

GGT $\rightarrow$ GAT $^{\mathrm{b}}($ Gly $\rightarrow$ Asp $)$ $\mathrm{CCT} \rightarrow$ CGT $($ Pro $\rightarrow$ Arg) 


\begin{tabular}{lcc}
\hline \multicolumn{1}{c}{ Exon/Intron } & Codon & Codon Change \\
\hline Variants of Unknown Significance & nucl 94 \\
1 & 295 & C $\rightarrow$ CCC $\rightarrow$ CTC \\
6 & 1094 & GTG $\rightarrow$ GCG \\
19
\end{tabular}

${ }^{a}$ Mutation listed in PTCH Mutation Database (includes mutations found in tumors) at as of May 8, 2005.

${ }^{b}$ Mutation R135X in exon 3 was found in four pedigrees.

'In-frame deletions, duplications, and missense mutations were judged deleterious if they were not found in 100 unaffected individuals, altered highly conserved base pairs, and segregated with the disease in those cases for which more than one affected family member was available for study.

${ }^{d}$ Changes in invariant bases (AG preceding exons and GT following exons) were assumed to affect splicing. All other changes were predicted to affect splicing by the splice site predictor programs at http://www.fruitfly.org or http://125.itba.mi.cnr.it/ webgene/wwwspliceview.html. The mutation in the last base of exon was assumed to affect splicing based on the preceding criteria.

${ }^{e}$ Mutation is in the last nucleotide of exon 10.

Table 3

Mutations by exon ${ }^{a}$

\begin{tabular}{|c|c|c|}
\hline Exon & Number & Size (bp) \\
\hline 1 & 0 & 189 \\
\hline $1 \mathrm{~A}$ & 0 & 185 \\
\hline 1B & 0 & 201 \\
\hline 2 & 6 & 193 \\
\hline 3 & 1 & 190 \\
\hline 4 & 0 & 70 \\
\hline 5 & 2 & 92 \\
\hline 6 & 5 & 199 \\
\hline 7 & 2 & 122 \\
\hline 8 & 3 & 148 \\
\hline 9 & 2 & 132 \\
\hline 10 & 3 & 156 \\
\hline 11 & 1 & 99 \\
\hline 12 & 1 & 126 \\
\hline 13 & 2 & 119 \\
\hline 14 & 0 & 403 \\
\hline 15 & 3 & 310 \\
\hline 16 & 2 & 143 \\
\hline 17 & 2 & 184 \\
\hline 18 & 0 & 281 \\
\hline 19 & 2 & 138 \\
\hline 20 & 2 & 143 \\
\hline 21 & 1 & 100 \\
\hline 22 & 0 & 255 \\
\hline 23 & 0 & 540 \\
\hline
\end{tabular}

${ }^{a}$ Variants of unknown significance not included.

mutations. Three pedigrees for which clinical information was available had variants of unknown significance. Because some NBCCS features have age-dependent expression, the ages of the patients in our test population are relevant to a phenotypic analysis. For example, children are much less likely to develop basal cell carcinomas than adults, and the frequency of occurrence of these neoplasms is highly correlated with age. The ages of the members of the pedigrees who were tested for $P T C H$ mutations ranged from newborn to 61 years. Their mean and median ages were $23.0(\sigma=16.4)$ and 18.5 years, respectively. The ages of the positive members of these families ranged from newborn to 45 years, with mean and median ages of $18.0(\sigma=$ 13.3 ) and 16.5 years, respectively. Patients testing negative for PTCH mutations ranged from 2 to 61 years of age. The mean and median ages of these latter patients were slightly higher than patients in whom $P T C H$ mutations were identified (25.2 $[\sigma=17.7]$ and 25.5 years, respectively) $(P=.03)$.

Forty-two patients were classified as sporadic, on the basis of affirmatively reported negative family history or tests performed on parents. Twenty-three of these patients tested positive for $P T C H$ mutations (54.8\%). Conversely, of the PTCH positive pedigrees, 23 of 47 consisted of a single sporadic patient $(48.9 \%)$. This proportion is consistent with published estimates of the frequency of new mutations in NBCCS cases of approximately $50 \% .^{1}$

One pedigree consisted of two affected siblings, each of whom had the same 21 base pair deletion in exon 9 of PTCH. The deletion was not found in peripheral blood leukocyte DNA of either parent, indicating that one parent is a gonadal mosaic for the mutation. Gonadal mosaicism for a $\mathrm{PTCH}$ mutation has been reported in NBCCS. ${ }^{9}$ Although it is not common, this finding indicates that siblings of sporadic patients still carry some risk of having the syndrome.

Among pedigrees testing positive for $\mathrm{PTCH}$ mutations, all had more than one typical NBCCS characteristic that was included in our questionnaire, and 23 of 28 positive pedigrees had three or more typical features of the disease (Table 4). Conversely, the pedigrees most likely to test positive for $\mathrm{PTCH}$ mutations had multiple features of NBCCS. Twenty-seven of 46 pedigrees (58.7\%) with two or more typical radiographic or pathologic features of NBCCS tested positive for $\mathrm{PTCH}$ mutations.

Jaw cysts were reported in most of the $\mathrm{PTCH}$-positive pedigrees that we tested. Of the 28 positive pedigrees, 26 had jaw cysts in combination with other features of the syndrome (Tables 5 and 6). Both of the two positive pedigrees without jaw cysts consisted of sporadically affected young children who 
Table 4

Number of clinical features in $\mathrm{PTCH}$ positive pedigrees

\begin{tabular}{cc}
\hline $\begin{array}{c}\text { Number of } \\
\text { Features }\end{array}$ & Proportion \\
\hline 2 & $5 / 28$ \\
3 & $8 / 28^{a}$ \\
4 & $7 / 28^{a}$ \\
5 & $4 / 28$ \\
6 & $4 / 28$
\end{tabular}

${ }^{a}$ One pedigree without jaw keratocysts.

Table 5

Frequency of clinical features in $\mathrm{PTCH}$ positive pedigrees

\begin{tabular}{lcc}
\hline Clinical Feature & Number & Fraction Positive \\
\hline JK & 26 & $26 / 28(92.9 \%)$ \\
PP & 17 & $17 / 28(60.7 \%)$ \\
SKELABN & 16 & $16 / 28(57.1 \%)$ \\
BCCs & 12 & $12 / 28(42.9 \%)$ \\
OCABN & 12 & $12 / 28(42.9 \%)$ \\
CLP & 5 & $5 / 28(17.9 \%)$ \\
CFC & 4 & $4 / 28(14.3 \%)$ \\
CF & 4 & $4 / 28(14.3 \%)$ \\
ACC & 3 & $3 / 28(10.7 \%)$ \\
OFMBOMA & 32 & $3 / 28(10.7 \%) 2 / 28(7.1 \%)$ \\
POLYD & 2 & $2 / 28(7.1 \%)$ \\
\hline
\end{tabular}

BCC, basal cell carcinoma; JK, jaw keratocysts; PP, palmar or plantar pit; CFC, calcification of the falx cerebri; MBOMA, medulloblastoma; SKELABN, skeletal abnormality; POLYD, polydactyly; OF, ovarian fibroma; $\mathrm{CF}$, cardiac fibroma; OCABN, ocular abnormality; ACC, agenesis of the corpus callosum or other CNS abnormalities; CLP, cleft lip or palate.

Table 6

Phenotypes in pedigrees and sporadic patients tested for $\mathrm{PTCH}$ mutations

\begin{tabular}{lcl}
\hline \multicolumn{1}{c}{$\begin{array}{c}\text { Combination } \\
\text { of Features }\end{array}$} & $\begin{array}{c}\text { Proportion } \\
\text { Testing Positive }\end{array}$ & $\begin{array}{c}\text { Statistical } \\
\text { Significance }\end{array}$ \\
\hline JK, PP +/- others & $17 / 21$ & \\
JK, PP without BCCs $+/-$ others & $9 / 11$ & $\mathrm{P}=0.36$ \\
JK, PP, BCCs, +/- others & $8 / 10$ & $\mathrm{P}=0.37$ \\
JK, BCCs, without PP +/- others & $4 / 6$ & $\mathrm{P}=0.30$ \\
JK without BCCs or PP +/- others & $5 / 11$ & $\mathrm{P}=0.04$ \\
BCCs, PP without JK +/- others & $0 / 8$ & $\mathrm{P}=0.0001$ \\
JK alone & $0 / 4$ & $\mathrm{P}=0.006$ \\
BCCs alone & $0 / 13$ & $\mathrm{P}=<0.0001$ \\
\hline
\end{tabular}

BCC, basal cell carcinoma; JK, jaw keratocysts; PP, palmar or plantar pit.

would not yet have been expected to develop this manifestation, but had at least three other features of the syndrome. Nevertheless, it should be noted that $P T C H$ mutations were not found in any of the four pedigrees with jaw cysts alone.
In regard to individual NBCCS features, 26 of 38 pedigrees with jaw cysts tested positive for PTCH mutations (68.4\%), whereas mutations were found in 17 of 30 with palmar pits (56.7\%), 4 of 10 with calcification of the falx cerebri (40.0\%), only 12 of 44 with basal cell carcinomas (27.3\%), 16 of 22 with skeletal anomalies $(72.3 \%), 2$ of 12 with medulloblastoma (16.7\%), and 12 of 14 with ocular abnormalities (85.7\%). In addition, 2 of 2 pedigrees with polydactyly (100\%), 3 of 7 with ovarian fibroma (42.9\%), 4 of 4 with cardiac fibroma (100\%), 3 of 5 with agenesis of the corpus callosum or other central nervous system abnormalities (60.0\%), and 5 of 5 with cleft lip and palate (100\%) had PTCH mutations. However, it is critical to note the limited predictive value of any single feature of NBCCS.

Of the PTCH-positive pedigrees only 12 of 28 (42.9\%) reported having basal cell carcinomas (Table 5), whereas 31 of 44 (70.5\%) of the PTCH-negative pedigrees had basal cell carcinomas. When 13 pedigrees that had basal cell carcinomas without other features of NBCCS (see below) were excluded from this analysis, 18 of 31 (58.1\%) of the $P T C H$-negative pedigrees had basal cell carcinomas. The relatively limited proportion of $P T C H$-positive families with basal cell carcinomas is a reflection of the age of our test population, because many individuals were tested before basal cell carcinomas were likely to have developed. The high proportion of basal cell carcinomas in the $\mathrm{PTCH}$-negative pedigrees is largely the result of referrals of patients with basal cell carcinomas who lack other features of NBCCS.

Notably, none of the 13 pedigrees affected by multiple or early-onset basal cell carcinomas without other manifestations of NBCCS tested positive for PTCH mutations. (One pedigree with only basal cell carcinomas consisted of a sole patient in whom a variant of unknown significance was found.) Extreme phenotypes were found among members of these pedigrees, including 22- and 26-year-old patients with 40 and 60 basal cell carcinomas, respectively, a 53-year-old woman who had developed 200 basal cell carcinomas, and a 61-year-old man with 100 basal cell carcinomas (Fig. 1).

\section{DISCUSSION}

Molecular testing for $\mathrm{PTCH}$ mutations can be an important diagnostic tool in the evaluation of patients at risk for or suspected of having NBCCS. The age-related penetrance of many NBCCS features makes DNA-based testing especially useful for young children. Physical examination is likely to be unrevealing in children aged less than 10 years, and it is unlikely that the combination of physical examination plus diagnostic $\mathrm{X}$-ray films is more cost-effective than DNA-based testing.

Surveillance recommendations for patients with NBCCS include semiannual neurologic evaluation and annual magnetic resonance imaging of the head to detect medulloblastoma until age 8 years; dermatologic assessment for basal cell carcinomas from early childhood through adulthood; echocardiography for at-risk babies at birth and on clinical suspicion of cardiac fibroma; regular dental surveillance for jaw cysts from early 


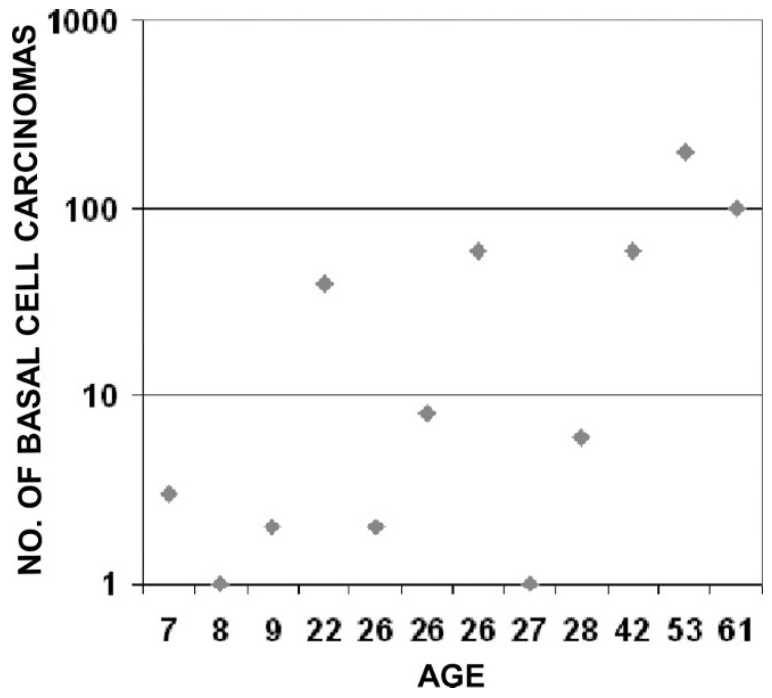

Fig. 1. Age versus number of basal cell carcinomas in nevoid basal cell carcinoma (NBCCS) syndrome-negative, basal cell carcinoma only patients. Extreme phenotypes were found among NBCCS-negative pedigrees with only basal cell carcinomas.

childhood; and initial pelvic ultrasound for ovarian fibromas at the preteen stage with repeated examination if a patient becomes symptomatic. Patients should be advised to reduce ultraviolet light exposure because this may lessen the risk of basal cell carcinoma development. Radiation therapy should also be avoided if possible because of the dramatically increased risk of basal cell carcinomas and the heightened risk of formation of other tumors in the radiation fields. ${ }^{2,4,23}$ A negative genetic test will spare at-risk patients from families with known mutations these costly and potentially traumatic surveillance and preventive measures.

Approximately $40 \%$ of the 106 pedigrees tested in this study were found to have $\mathrm{PTCH}$ mutations. The relatively low percentage of $\mathrm{PTCH}$-positive pedigrees appears to reflect the submission for testing of patients from pedigrees with variant phenotypes. Applying the NBCCS diagnostic criteria set forth by Evans and Farndon ${ }^{23}$ to our test pedigrees, 25 of 39 (64.1\%) pedigrees that met these criteria for NBCCS tested positive for PTCH mutations. Conversely, only 3 of 33 (9.0\%) of pedigrees that would not be classified as having NBCCS using these criteria tested positive for $\mathrm{PTCH}$ mutations. All three such pedigrees consisted of sporadic patients. Two of these patients were newborns with multiple features of NBCCS, and the third was a 17 year old with multiple skeletal anomalies and jaw keratocysts. Results using the diagnostic criteria of Kimonis et al. ${ }^{4}$ were similar, with 23 of $38(60.5 \%)$ of the pedigrees meeting these criteria testing positive for $\mathrm{PTCH}$ mutations and only 5 of $37(13.5 \%)$ pedigrees that would not be classified as having NBCCS testing positive for $\mathrm{PTCH}$ mutations.

Patients with pedigrees that displayed the classic features of NBCCS, but in whom we were unable to detect mutations, may have large deletions or other gross rearrangements that would not be detected by sequencing, or mutations in functionally important regions of the gene that lie outside the target sequences of our primer sets. For example, a putative fourth alternate splice form of exon 1 has recently been described. ${ }^{24}$ Although large deletions encompassing PTCH have been seen, reported investigations suggest that they are extremely uncommon among patients with typical NBCCS. ${ }^{7-9}$

Previously published mutational analyses of $\mathrm{PTCH}$ in patients with NBCCS have described a broad mutational spectrum among the $\mathrm{PTCH}$-positive pedigrees, with most families having unique mutations. Most described mutations have been predicted to produce truncated proteins, and few missense mutations have been described. ${ }^{9,21,25,26}$ Our data are consistent with earlier reports.

In vitro functional studies of a mouse $P T C$ protein containing a mutation analogous to G509V that were performed in a vertebrate cell line $\left(\mathrm{PZA}^{\mathrm{ptc}-l-}\right)$ demonstrated significantly reduced pathway inhibition relative to the wild-type PTC. ${ }^{18}$ In comparison, a mutation equivalent to G509V in Drosophila appears to act in a dominant-negative manner. However, this effect was not observed with another mutation in the same codon G509R, indicating that removal of the glycine residue alone is insufficient to produce dominant-negative function..$^{22}$ The apparent differences between the effect of the G509R mutation in fly and vertebrate systems may truly reflect a fundamental difference in PTC signaling between Drosophila and vertebrates, or may be an artifact of the experimental methods used. In either event, both studies support the pathogenicity of the G509V mutation and indicate a key role for this codon in $\mathrm{PTCH}$ functioning.

In our study, the presence of basal cell carcinomas was a relatively weak predictor of a positive test. This result is probably reflective of the age of our test population and clinician suspicion for NBCCS in patients with basal cell carcinomas and few or no other features of the syndrome. Of the 20 positive patients without basal cell carcinomas, 15 were aged less than 20 years (median age $=9.5$ years; mean age $=13.9$ years) . The mean age of the $12 \mathrm{PTCH}$-positive patients with basal cell carcinomas was 24.6 years, with a median of 25.5 years. One half of these patients were more than 30 years old.

Notably, none of the 13 pedigrees affected by multiple or early-onset basal cell carcinomas without other manifestations of NBCCS tested positive for PTCH mutations. (One pedigree with only basal cell carcinomas consisted of a sole patient in whom a variant of unknown significance was identified.) Extreme phenotypes were found among members of these pedigrees (Fig. 1), including 22- and 26-year-old patients with 40 and 60 basal cell carcinomas, respectively, a 53-year-old woman who had developed 200 basal cell carcinomas, and a 61-year-old man with 100 basal cell carcinomas.

It is difficult to explain the presentations of these pedigrees by fair skin and excessive sunlight exposure alone. The failure to find $P T C H$ mutations in these patients could be the result of large $P T C H$ deletions or transversions that would not be detected by sequencing, or mutations in functionally important intronic or noncoding regions that would not be identified by current test methods. Further, these patients may be mosaics, having $\mathrm{PTCH}$ mutations in the tumor-forming skin, but not in peripheral blood leukocytes. Mosaicism could be studied by 
analyzing the skin adjacent to basal cell carcinomas for $\mathrm{PTCH}$ mutations. A third explanation is that the patients have other syndromes associated with basal cell carcinomas, for example, Bazex-Dupre-Christol syndrome or Rombo syndrome, an autosomal dominant disorder characterized by vermiculate atrophoderma, milia, hypotrichosis, trichoepitheliomas, basal cell carcinomas, and peripheral vasodilation with cyanosis. ${ }^{27}$ These syndromes can probably be distinguished clinically. Fourth, these $\mathrm{PTCH}$-negative families may have mutations elsewhere in the Hedgehog pathway, for example, sporadic basal cell carcinomas that lack PTCH mutations often have smoothened mutations. Finally, it is possible that some of these kindreds have mutations in other cancer predisposition genes. Allelic loss of 4q, 1q, 15q, 17p, and 18q, among others, have been reported in some basal cell carcinomas, ${ }^{10,12,28,29}$ suggesting the presence of tumor suppressor genes in these regions. It is possible that germline mutations in these putative tumor suppressors underlie some cases of nonsyndromic hereditary basal cell carcinomas.

None of the eight pedigrees with the combination of basal cell carcinomas and palmar or plantar pits tested positive for PTCH mutations. This is surprising, because palmar pits are a frequent finding in NBCCS, and are relatively specific for the syndrome. Thus, one would expect to find $P T C H$ mutations in a high proportion of pedigrees with this constellation of findings. However, true palmar or plantar pits can be difficult to diagnose clinically. The authors suspect that these data may reflect misdiagnosis of palmar or plantar pits in pedigrees that have basal cell carcinomas without other manifestations of NBCCS. Lesions resembling pits may occur in creases of normal palms, but these lesions lack characteristics of the pits in NBCCS. In particular, NBCCS-related pits are found in regions between creases and sometimes in the webbing between fingers. Characteristic of these pits is a red base in light-skinned people, and a dark brown or black base in heavily pigmented people. They are usually sand-grained size or larger.

This review represents an analysis of the characteristics and test results of all patients referred to our laboratory for clinical NBCCS testing during the study period. Although this method of ascertainment did not necessarily introduce bias into our results, we were more likely to obtain clinical information about $P T C H$-negative pedigrees than those that tested positive. This discrepancy may reflect the reluctance of providers who were retrospectively questioned to share clinical information about pedigrees with known disease-causing mutations, presumably because of privacy concerns. Another potential source of bias is created by the cost of $\mathrm{PTCH}$ mutation testing and a lack of insurance coverage for the testing among many patients. This could result in the testing of a greater proportion of nonobvious or ambiguous cases or children than is reflected in the actual population of patients with NBCCS. Also, the high prevalence of jaw cysts among $\mathrm{PTCH}$-positive pedigrees may be a consequence of ascertainment bias. The presence of jaw cysts in an affected patient may have made it more likely that NBCCS was recognized. This could have resulted in more accurate clinical diagnoses in such patients and the dispropor- tionate representation of jaw cysts within our $\mathrm{PTCH}$-positive pedigrees.

Because our data are derived from patients whose blood samples were sent for clinical testing, as opposed to research subjects, we had little influence over the amount or quality of clinical information that we received about each pedigree. The lack of standardized clinical assessment of patients and pedigrees may have led to some inaccuracy in our data. For example, because our patients and pedigrees were not systematically evaluated in the way that they would have been assessed in a prospective study, it is likely that less easily recognized or less commonly evaluated features of NBCCS, such as calcification of the falx cerebri, ovarian fibromas, or even palmar pits, were understated among $\mathrm{PTCH}$-positive patients.

Despite these limitations, the information we present has definite value for clinicians as a reflection of real-world experience. Although our data in some instances may have been limited, this is also true for clinicians who often receive incomplete family histories and frequently lack opportunities to systematically assess multiple family members of patients in whom they are considering the diagnosis of NBCCS.

\section{ACKNOWLEDGMENTS}

This work was supported by National Institutes of Health grant R01CA93908 to A.E.B. and by 5T32GM008753, which supported Dr. Klein. We thank Patricia Ann Beck and Devon Pleau for their assistance with this project, the other members of the Bale Laboratory, and especially the many patients, physicians, and counselors who have entrusted our laboratory with their clinical NBCCS testing.

\section{References}

1. Gorlin RJ. Nevoid basal cell carcinoma (Gorlin) syndrome. Genet Med 2004;6:530 538.

2. Evans DG, Ladusans EJ, Rimmer S, Burnell LD, Thakker N, Farndon PA. Complications of the naevoid basal cell carcinoma syndrome: results of a population based study. J Med Genet 1993;30:460-464.

3. Shanley S, Ratcliffe J, Hockey A, Haan E, et al. Nevoid basal cell carcinoma syndrome: review of 118 affected individuals. Am J Med Genet 1994;50:282-290.

4. Kimonis VE, Goldstein AM, Pastakia B, Yang ML, et al. Clinical manifestations in 105 persons with nevoid basal cell carcinoma syndrome. Am J Med Genet 1997;69: 299-308.

5. Kimonis VE, Mehta SG, DiGiovanna JJ, Bale SJ, Pastakia B. Radiological features in 82 patients with nevoid basal cell carcinoma (NBCC or Gorlin) syndrome. Genet Med 2004;6:495-502.

6. Evans DG, Farndon PA, Burnell LD, Gattamaneni HR, Birch JM. The incidence of NBCCS in 173 consecutive cases of medulloblastoma. Br J Cancer 1991;64:959-961.

7. Hahn H, Wicking C, Zaphiropoulos PG, Gailani MR, et al. Mutations of the human homolog of Drosophila patched in the nevoid basal cell carcinoma syndrome. Cell 1996;85:841-851.

8. Johnson RL, Rothman AL, Xie J, Goodrich LV, et al. Human homolog of patched, a candidate gene for the basal cell nevus syndrome. Science 1996;272:1668-1671.

9. Wicking C, Shanley S, Smyth I, Gillies S, et al. Most germ-line mutations in the nevoid basal cell carcinoma syndrome lead to a premature termination of the PATCHED protein, and no genotype-phenotype correlations are evident. Am J Hum Genet 1997;60:21-26.

10. Gailani MR, Bale SJ, Leffell DJ, DiGiovanna JJ, et al. Developmental defects in NBCCS related to a putative tumor suppressor gene on chromosome 9. Cell 1992; 69:111-117.

11. Gailani MR, Stahle-Backdahl M, Leffell DJ, Zaphiropoulos PG, et al. The role of the human homologue of Drosophila patched in sporadic basal cell carcinomas. Nat Genet 1996;14:78-81. 
12. Gailani MR, Leffell DJ, Ziegler A, Gross EG, Brash DE, Bale AE. relationship between sunlight exposure and a key genetic alteration in basal cell carcinoma. J Natl Cancer Inst 1996;88:349-354

13. Unden AB, Holmberg E, Lundh-Rozell B, Stahle-Backdahl M, et al. Mutations in the human homologue of Drosophila patched $(\mathrm{PTCH})$ in basal cell carcinomas and the NBCCS: different in vivo mechanisms of PTCH inactivation. Cancer Res 1996;56: 4562-4565.

14. Levanat S, Gorlin RJ, Fallet S, Johnson DR, Fantasia JE, Bale AE. A two-hit model for developmental defects in NBCCS. Nat Genet 1996;12:85-87.

15. Cohen MM. The hedgehog signaling network. Am J Med Genet 2003; 123A: 5-28.

16. MarigoV, Davey RA, Zuo Y, Cunningham JM, Tabin CJ. Biochemical evidence that patched is the Hedgehog receptor. Nature 1996;384:176-179.

17. Stone DM, Hynes M, Armanini M, Swanson TA, et al. The tumour-suppressor gene patched encodes a candidate receptor for Sonic hedgehog. Nature 1996;384:129 134.

18. Taipale J, Cooper MK, Maiti T, Beachy PA. Patched acts catalytically to suppress the activity of Smoothened. Nature 2002;418:892-897.

19. Ciulla TA, Sklar RM, Hauser SL. A simple method for DNA purification from peripheral blood. Anal Biochem 1988;174:485-488.

20. Jeanpierre M. A rapid method for the purification of DNA from blood. Nucleic Acids Res 1987;15:9611.

21. Fujii K, Kohno Y, Sugita K, Nakamura M, et al. Mutations in the human homologue of Drosophila patched in Japanese nevoid basal cell carcinoma syndrome patients. Hum Mutat 2003;21:451-452.
22. Hime GR, Lada H, Fietz MJ, Gillies S, et al. Functional analysis in drosophila indicates that the NBCCS/PTCH1 mutation G509V results in activation of smoothened through a dominant-negative mechanism. Dev Dyn 2004;229:780-790.

23. Evans DG, Nevoid basal cell carcinoma syndrome. GeneReviews [Internet database]. October 6, 2004. Available at: http://www.genetests.org. Accessed May 2005.

24. Nagao K, Toyoda M, Takeuchi-Inoue K, Fujii K, Yamada M, Miyashita T. Identification and characterization of multiple isoforms of a murine and human tumor suppressor, patched, having distinct first exons. Genomics 2005;85:462-471.

25. Chidambaram A, Goldstein AM, Gailani MR, Gerrard B, et al. Mutations in the human homologue of the Drosophila patched gene in Caucasian and African-American nevoid basal cell carcinoma syndrome patients. Cancer Res 1996;56:4599-4601.

26. Boutet N, Bignon Y, Drouin-Garraud V, Sarda P, et al. Spectrum of PTCH1 mutations in French patients with Gorlin syndrome. J Invest Dermatol 2003;121:478-481.

27. Michaelsson G, Olsson E, Westermark P. The Rombo syndrome: a familial disorder with vermiculate atrophoderma, milia, hypotrichosis, trichoepitheliomas, basal cell carcinomas and peripheral vasodilation with cyanosis. Acta Derm Venereol 1981;61:497-503

28. Cho S, Hahm JH, Hong YS. Analysis of $p 53$ and $B A X$ mutations, loss of heterozygosity, $p 53$ and $B C L 2$ expression and apoptosis in basal cell carcinoma in Korean patients. Br J Dermatol 2001;144:841-848.

29. Sironi E, Cerri A, Tomasini D, Sirchia SM, et al. Loss of heterozygosity on chromosome 4q32-35 in sporadic basal cell carcinomas: evidence for involvement of p33ING2/ING1L and SAP30 genes. J Cutan Pathol 2004;31:318-322. 